\title{
Influence of Drug Properties and Formulation on In Vitro Drug Release and Biowaiver Regulation of Oral Extended Release Dosage Forms
}

\author{
Zhongqiang Lin, ${ }^{1,2}$ Deliang Zhou, ${ }^{1}$ Stephen Hoag, ${ }^{2}$ and Yihong Qiu ${ }^{1,3}$
}

Received 13 October 2015; accepted 14 December 2015; published online 14 January 2016

\begin{abstract}
Bioequivalence (BE) studies are often required to ensure therapeutic equivalence for major product and manufacturing changes. Waiver of a BE study (biowaiver) is highly desired for such changes. Current regulatory guidelines allow for biowaiver of proportionally similar lower strengths of an extended release (ER) product provided it exhibits similar dissolution to the higher strength in multimedia. The objective of this study is to demonstrate that (1) proportionally similar strengths of ER tablets exhibiting similar in vitro dissolution profiles do not always assure BE and (2) different strengths that do not meet the criteria for dissolution profile similarity may still be bioequivalent. Four marketed ER tablets were used as model drug products. Higher and lower (half) strength tablets were prepared or obtained from commercial source. In vitro drug release was compared using multi-pH media $(\mathrm{pH} \mathrm{1.2,} \mathrm{4.5,}$ 6.8) per regulatory guidance. In vivo performance was assessed based on the available in vivo BE data or established in vitro-in vivo relationships. This study demonstrated that the relationship between in vitro dissolution and in vivo performance is complex and dependent on the characteristics of specific drug molecules, product design, and in vitro test conditions. As a result, proportionally similar strengths of ER dosage forms that meet biowaiver requirements per current regulatory guidelines cannot ensure bioequivalence in all cases. Thus, without an established relationship between in vitro and in vivo performance, granting biowaiver based on passing in vitro tests may result in the approval of certain bioinequivalent products, presenting risks to patients. To justify any biowaiver using in vitro test, it is essential to understand the effects of drug properties, formulation design, product characteristics, test method, and its in vivo relevance. Therefore, biowaiver requirements of different strengths of ER dosage forms specified in the current regulatory guidance should be reevaluated to assure consistent safety and efficacy among different strengths.
\end{abstract}

KEY WORDS: bioequivalence; biowaiver; extended release; in vitro drug release; in vivo evaluations; proportionally similar strength.

\section{INTRODUCTION}

In vitro drug release is one of the most critical quality attributes currently used for product quality assessment and process control, for assuring sameness after making product/ process changes and for supporting waiver of in vivo bioequivalence studies (biowaiver) under certain conditions. Over the last two decades, in vitro release and its application in supporting biowaivers have received increased attention from the regulators, industry, and academia, particularly for products with release rate-limited absorption, such as extended release (ER)

\footnotetext{
${ }^{1}$ Oral Drug Products, Manufacturing Science and Technology, AbbVie, Inc., Dept -045M, Bldg A4-2, 1401 Sheridan Road, North Chicago, Illinois 60064-6235, USA.

${ }^{2}$ School of Pharmacy, University of Maryland, Baltimore, Maryland, USA.

${ }^{3}$ To whom correspondence should be addressed. (e-mail: Qiu.yihong@abbvie.com)
}

dosage forms, because of its likely link to in vivo performance. For oral ER drug products, in vitro drug release test can be used as a surrogate for in vivo bioequivalence study when an in vitroin vivo correlation (IVIVC) is established and validated according to the regulatory guidance issued by the US Food and Drug Administration (FDA) and European Medicine Agency (EMA) (1-3). Current regulation also allows the use of in vitro release test to support biowaiver between the proportionally similar higher and lower strengths of the same drug product regardless of the type of ER dosage forms or whether there is a qualitative or quantitative in vitro-in vivo relationship (IVIVR). As a result, the lower strengths and, in some cases, higher strengths of many oral ER products have been approved without conducting bioequivalence studies.

Oral ER products are generally single-unit or multipleunit dosage forms (pellets, tablets, or capsules) that involve one or more of the drug release mechanisms, such as diffusion, erosion, dissolution, and osmotic pressure (4). It is well established in the scientific field that in vitro drug release from an ER system usually depends on drug properties, type 
of delivery technology, formulation composition, size and geometry of the dosage form, manufacturing process as well as test method and conditions. However, how the abovementioned variables and their interplays may impact the association between in vitro drug release in multimedia and in vivo absorption has not been well understood. More importantly, a quantitative link between the in vitro and in vivo performance that can be generalized for any types of drugs and dosage forms does not exist. As a result, it is often challenging to determine whether or how a change in dissolution observed during product development, postapproval changes, or routine commercial production may impact in vivo performance in the absence of an IVIVR. For example, a formulation or manufacturing change that results in passing or failing similarity factor $\left(f_{2}\right)$ criteria may or may not have direct in vivo implication depending on the characteristics of the active pharmaceutical ingredient (API) and product design, the nature of the change, and the test method. Except for the multiple-unit dosage forms where various strengths differ only in the number of units, a lower strength of an ER drug product made either by making proportional change in composition or by only matching in vitro drug release may or may not be bioequivalent to the higher strength. Hence, allowing biowaiver for lower strengths of ER products based on in vitro drug release in the absence of an IVIVC or IVIVR by current regulatory guidelines can result in compromised product performance and increased risks to the patients. This is of particular concern for products containing APIs with narrow therapeutic index (NTI). Therefore, an improved understanding of the drug release in relation to API, drug product, and test method is urgently needed to provide sound scientific basis for regulatory requirements of biowaiver and to assure product quality.

In the present study, the in vitro release of proportionally similar higher and lower strengths of ER tablets was evaluated and compared with the corresponding in vivo performance. Four model drug products, namely extended release tablets of drug A, drug B, drug $\mathrm{C}$, and verapamil hydrochloride, were chosen to show four possible scenarios illustrated in Fig. 1. The findings can have significant scientific, regulatory, and business implications with respect to regulatory clearance of proportionally similar strengths of an ER product that are only required to exhibit similar in vitro drug release in multimedia.

\section{EXPERIMENTAL SECTION}

\section{Materials and Equipment}

The following materials and equipment were used in the study: compound A (AbbVie, North Chicago, IL), compound B (AbbVie, North Chicago, IL), compound C (AbbVie, North Chicago, IL), Isoptin ${ }^{\circledR}$ SR tablets (120 mg Lot \#316168D, $240 \mathrm{mg}$ Lot \#286628D; Abbott Laboratories, Limited, Québec, Canada), Methocel K15MP CR (Dow Chemical Co., Midland, MI), Methocel E10M (Dow Chemical Co., Midland, MI), and Methocel K100LV CR (Colorcon, West Point, PA).
Equipment used were as follows: KG5 high shear granulator (Key International, Englishtown, NJ), Fitzpatrick L1A Comminutor (The Fitzpatrick Company, Elmhurst, IL), Strea-1 ${ }^{\mathrm{TM}}$ fluid bed dryer (Aeromatic-Fielder AG, Bubendorf, Switzerland), Twin Shell Dry Blender (Patterson-Kelley Co., East Stroudsburg, PA), Carver laboratory press (Model: 88881DI0A00, Carver, INC Wabash, IN), VK7000 dissolution testing stations (Varian Inc., Palo Alto, CA), and Perkin Elmer 200 HPLC (Waltham, MA).

\section{Methods}

\section{Preparation of Tablets}

Low and high strengths of Isoptin ${ }^{\circledR}$ SR tablets that are proportionally similar in composition were obtained from commercial source. Proportionally similar low and high strengths of extended release tablets of drug A, drug $\mathrm{B}$, and drug $\mathrm{C}$ were prepared by using the same formulations and manufacturing processes of the corresponding commercial tablets, drug A ER, drug B ER, and drug C ER tablets, respectively. All three products were made using wet granulation in a high shear granulator followed by fluid bed drying, milling, blending, and compression.

\section{Evaluation of Tablets}

In the present study, the higher strength of the marketed dosage form was used as a reference for the evaluation of in vitro and in vivo performance of the proportionally similar lower strength of each product. Specifically, extended release tablets of drug A (500 mg), drug B (500 mg), drug C (1000 $\mathrm{mg}$ ), and verapamil hydrochloride $(240 \mathrm{mg}$ ) were compared with the corresponding half-strength tablets, 250, 250,500 , and $120 \mathrm{mg}$, respectively.

\section{Evaluation of In Vitro Drug Release}

In vitro dissolution experiments were carried out for all four drug products using USP Apparatus I or II in various media at $37.0 \pm 0.5^{\circ} \mathrm{C}$. Samples were taken at predetermined time intervals for analysis. Each product was tested using the corresponding NDA and/or USP test methods as well as multi-pH media.

ER Tablets of Drug A: The compendial method used USP II at $100 \mathrm{rpm}$ in $500 \mathrm{~mL}$ of $0.1 \mathrm{~N} \mathrm{HCl}$ for $45 \mathrm{~min}$ followed by $900 \mathrm{~mL}$ of $0.05 \mathrm{M}$ phosphate buffer containing $75 \mathrm{mM}$ sodium dodecyl sulfate (SDS), pH 5.5 with wire helical sinkers. This is an NDA test that allows the prediction of in vivo performance. The multimedia dissolution profiles were generated at $100 \mathrm{rpm}$ in $900 \mathrm{~mL} \mathrm{pH} \mathrm{1.2,} \mathrm{4.5,} \mathrm{and} 6.8$ buffers using wire helical sinkers.

ER Tablets of Drug B: The tablets were tested using USP Apparatus II at $50 \mathrm{rpm}$ in $900 \mathrm{~mL} 0.21 \mathrm{M}$ phosphate buffer at $\mathrm{pH} 4.0$ with wire helical sinkers. This test is used to relate the critical formulation variables to the in vitro and in vivo 


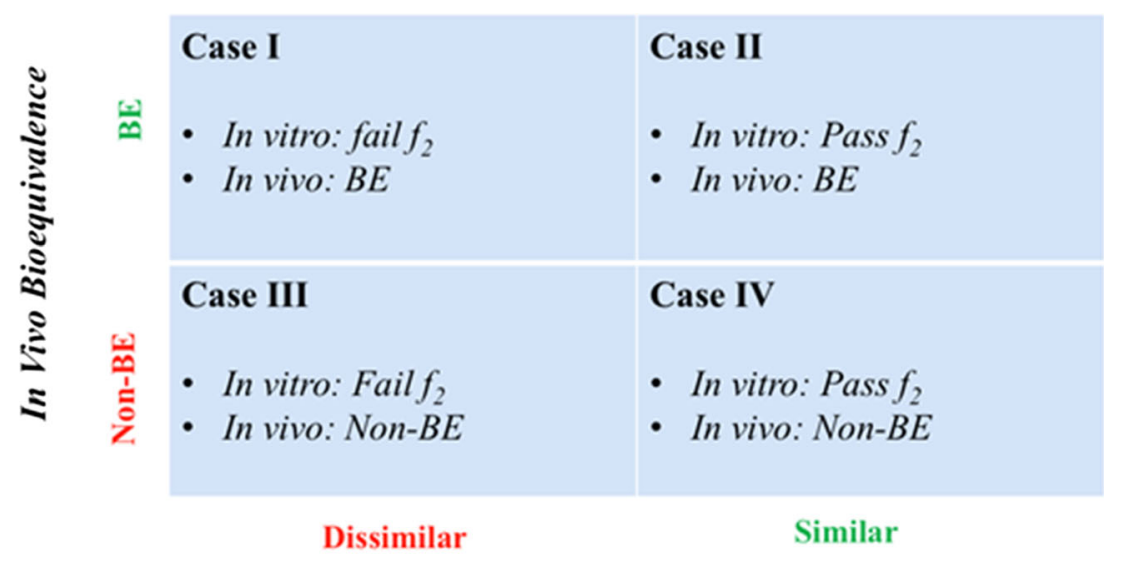

In Vitro Drug Release

Fig. 1. Four possible scenarios linking in vitro drug release to in vivo performance between the proportionally similar strengths of an ER product

performance. The multimedia dissolution profiles were generated at $50 \mathrm{rpm}$ in $900 \mathrm{~mL} \mathrm{pH} 4.5$ and 6.8 buffers using wire helical sinkers.

ER Tablets of Drug C: The compendial method used USP Apparatus I (40 mesh basket) at $100 \mathrm{rpm}$ in $900 \mathrm{~mL}$ of water. The multimedia dissolution profiles were generated at $100 \mathrm{rpm}$ in $900 \mathrm{~mL}$ pH 1.2, 4.5, and 6.8 buffers.

Isoptin ${ }^{\circledR}$ SR Tablets: The compendial method used USP Apparatus II at $50 \mathrm{rpm}$ in $900 \mathrm{~mL}$ of $\mathrm{pH} 1.0$ medium for $1 \mathrm{~h}$ followed by $900 \mathrm{~mL}$ of simulated intestinal fluid. The multimedia dissolution profiles were generated at $50 \mathrm{rpm}$ in $900 \mathrm{~mL}$ pH 1.2, 4.5, and 6.8 buffers.

The higher strengths were first tested using the method approved by the FDA to ensure that the in vitro performance is the same as the clinical batches used in the in vivo bioavailability studies during product development. Subsequently, drug release profiles of both the lower and higher strengths were generated using three USP buffer media at $\mathrm{pH} 1.2,4.5$, and 6.8 (5). When testing dissolution of drug A ER tablets at $\mathrm{pH} 1.2$ and drug B ER tablets at pH 6.8, two 250-mg tablets were used to compare with a single $500-\mathrm{mg}$ tablet, in order to match the effect of nonsink condition on drug release of the higher strength resulting from limited drug solubility under the testing conditions. To facilitate evaluation of their in vivo performance, in vitro dissolution of the lower and higher strengths was also tested using the IVIVC- or IVIVR-based methods that are available for drug $\mathrm{A}$ and drug B ER tablets, respectively.

All test samples were assayed for drug concentrations for each individual drug product using HPLC analytical methods described in USP-NF for each product (6-9). For every drug product, standard curves were constructed for each $\mathrm{pH}$ medium using a total of six concentrations and five replicates at each concentration. All assay methods showed good linearity (correlation coefficients > 0.999), accuracy, and reproducibility. Six or 12 replicates were tested to generate each profile. The drug release profiles of the higher and lower strengths were compared using the similarity factor $f_{2}$ (Eq. 1). The release profiles of the two strengths are considered similar if $f_{2} \geq 50$ and dissimilar if $f_{2}<50$.

$$
f_{2}=50 \times \log \left\{\left[1+\frac{1}{n} \sum_{j=1}^{n}\left|R_{j}-T_{j}\right|^{2}\right]^{-0.5} \times 100\right\}
$$

\section{In Vivo Evaluation}

The in vivo performances of the high and low strengths of the four drug products were evaluated and compared using data obtained from either bioequivalence studies or dissolution test based on an IVIVR or IVIVC model. Specifically, an IVIVC model validated internally and externally in bioavailability studies was used to evaluate the in vivo performance of drug A tablets (1). An in vitro-in vivo relationship based on single- and multiple-dose bioequivalence studies was used to assess the in vivo performance of drug B tablets (10), while the in vivo performances for drug $\mathrm{C}$ tablets $(11,12)$ and Isoptin ${ }^{\circledR}$ SR tablets $(13,14)$ were obtained from bioequivalence studies from literature.

ER Tablets of Drug A: The in vivo performance of extended release hydrophilic matrix tablets of drug A, 500 and $250 \mathrm{mg}$, was compared using a predictive in vitro dissolution method based on a validated IVIVC model. A total of five ER formulations with different release rates were used to establish and validate the linear IVIVC model (1). For internal validation, a single-dose four-way crossover study $(N=16)$ was performed using fast-, medium-, and slow- 
releasing ER formulations and a 12-h IV infusion as a reference (1). To validate the model externally, a second three-way crossover study $(N=36)$ was performed using slightly fast-, medium-, and slightly slow-releasing ER formulations (1). In the present study, bioequivalence between the 500- and 250-mg strengths were evaluated according to drug release specification limits established through convolution using the IVIVC model.

ER Tablets of Drug B: Comparison of the in vivo performance of extended release hydrophilic matrix tablets of drug $\mathrm{B}, 500$ and $250 \mathrm{mg}$, was made using a dissolution range with demonstrated bioequivalence. Three ER formulations (I, II, and III) containing $10 \%, 20 \%$, and $30 \%$ rate-controlling polymer, respectively, were manufactured using the same wet granulation process and tested in 24 healthy subjects (23 completed) in a four-way crossover single-dose bioavailability study using immediate release tablets as a reference (10). Formulations I, II, and III with a range of dissolution profiles were shown to be bioequivalent. The study results were confirmed in a multiple-dose three -way crossover bioavailability study of formulations I and II using IR tablets as reference (10) and were subsequently used to relate the critical formulation variables to the in vitro dissolution and in vivo bioavailability for defining a dissolution space in which bioequivalence can be assured. To compare the proportionally similar strengths of ER tablets of drug B in the present study, the two strengths were deemed to be bioequivalent when their dissolution profiles fall within the drug release space with proven bioequivalence.

ER Tablets of Drug C: Comparative in vivo bioavailability information of ER tablets of drug C, 500 and $1000 \mathrm{mg}$, was obtained from the results of bioequivalence studies reported in the literature $(11,12)$.

Isoptin $S R \circledast$ Tablets: In the present study, relative bioavailability studies of the 240- and 120-mg Isoptin SR® tablets available in the literature and regulatory filing documents were used to compare the in vivo performance of the two strengths.

\section{RESULTS}

For ER tablets of drugs A, B, and $\mathrm{C}$, the in vitro performance of the higher strength tablets prepared in this study was tested and confirmed to match that of the clinical batch of each product before being used as the reference for evaluation of the proportionally similar lower strengths. For Isoptin SR ${ }^{\circledR}$ tablets, both higher and lower strengths were commercial products obtained directly from the pharmacy.

\section{Comparing In Vitro and In Vivo Performances}

\section{ER Tablets of Drug A}

Dissolution profiles of the compositionally proportional lower strength drug A ER tablets $(250 \mathrm{mg}$ ) are compared with the higher strength $(500 \mathrm{mg})$ in Fig. 2. It was observed that the in vitro dissolution of drug A ER tablets varies significantly with $\mathrm{pH}$ due to $\mathrm{pH}$-dependent solubility and is inherently slow in the standard multi-pH media. Considering (i) gastric residence time of solid dosage forms is generally less than 2-4 $\mathrm{h}$ and (ii) complete in vivo drug release/ absorption of drug A ER tablets occurs within approximately $20 \mathrm{~h}$, test duration in all media was limited to $24 \mathrm{~h}$ for in vivo relevance and practical purpose. Data analysis shows that the lower strength meets the similarity factor $\left(f_{2}\right)$ criteria in all three media over the $\mathrm{pH}$ range of 1.2-6.8 using the conventional USP test method. Thus, according to the regulatory guidelines, a bioequivalence study for proportionally similar lower strength can be waived based on the following: both strengths (1) have the same dosage form design, (2) have the same drug release mechanism, and (3) are within the ranges of linear disposition and therapeutic dose.

However, the proportionally similar 250-mg strength was determined to be not bioequvalent to the $500 \mathrm{mg}$ based on the dissolution data generated using the IVIVC-based test method in Fig. 3. More specifically, the predicted $C_{\max }$ of the 250-mg tablets was found to be bioinequivalent to that of the 500-mg strength due to significantly faster drug release that was outside of the bioequivalent dissolution limits established by IVIVC. In fact, it was based on this finding that a decision was made to develop a new formulation for the 250-mg tablet in spite of the acceptable multimedia testing results of the proportionally similar formulation. Through formulation screening studies, the currently marketed 250-mg tablet was identified based on the bioequivalence prediction from the similar dissolution profile to the 500-mg strength when tested by the IVIVC-based dissolution method (Fig. 3). The reformulated $250-\mathrm{mg}$ commercial tablet represents a $50 \%$ increase and approximately $25 \%$ decrease in hydroxyl methylcellulose (HPMC) content and surface area/volume ratio $(\mathrm{S} / \mathrm{V})$, respectively, relative to the proportionally similar 250 -mg tablets. A subsequent single-dose pilot bioequivalence study in 24 healthy subjects not only further confirmed the IVIVC-based prediction but also inferred different in vivo performance of the proportionally similar formulation.

\section{ER Tablets of Drug B}

Figure 4 shows that dissolution profiles of the proportionally similar lower strength tablets $(250 \mathrm{mg})$ meet the similarity factor criteria in media over the $\mathrm{pH}$ range of 4.5-6.8 using conventional USP test method. Data in $\mathrm{pH} 1.2$ dissolution medium are unavailable because of the rapid degradation of drug $\mathrm{B}$ at low $\mathrm{pH}$ (15).

To compare the in vivo performance of the proportionally similar 250- and 500-mg strengths, a dissolution test that has shown the ability to map in vitro and in vivo performance of extended release hydrophilic matrix tablets of drug $B$ during product development was utilized. Specifically, a dissolution range with demonstrated bioequivalence of three ER formulations with differing levels of the rate-controlling polymer was established and confirmed in single-dose and multiple-dose crossover bioavailability studies (10) (Fig. 5a). In the present study, bioequivalence of the 250- and 500-mg strengths was concluded as the dissolution profiles of the two 

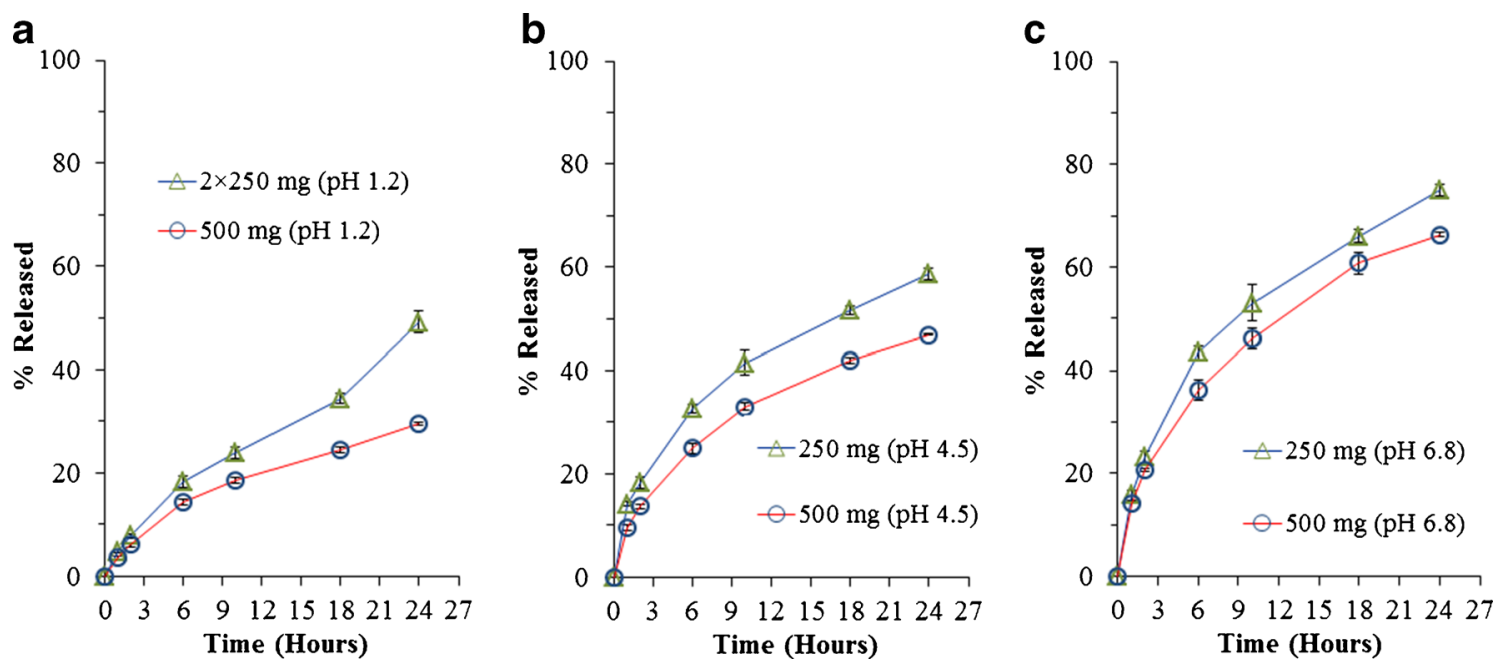

Fig. 2. In vitro dissolution of drug A ER tablets, 250 and $500 \mathrm{mg}$, in multi-pH media. a pH $1.2\left(f_{2}=51.2\right)$. b pH $4.5\left(f_{2}=54.1\right)$. c $\mathrm{pH} 6.8\left(f_{2}=61.0\right)$

strengths generated using the IVIVR-based test method shown in Fig. 5 b not only are similar $\left(f_{2}=61.0\right)$ but also fall well within the space with proven bioequivalence.

\section{ER Tablets of Drug C}

In vitro dissolution testing of the two proportionally similar strengths of drug C ER tablets (500 and $1000 \mathrm{mg}$ ) was carried out using a USP Apparatus I at different $\mathrm{pH}$ values of 1.2, 4.5, and 6.8. The data provided in Fig. 6 indicate dissimilar dissolution profiles under all test conditions except at $\mathrm{pH} 1.2$.

When the in vivo performance of the proportionally similar 500- and 1000-mg strengths of drug C ER tablets made by compressing the same granulation in different quantities was compared in bioavailability studies, the 1000- mg strength was found to be not bioequivalent with slower absorption. As a result, the two strengths are not interchangeable, and the 1000-mg strength of commercial ER tablet of drug $\mathrm{C}$ was later reformulated to achieve bioequivalence to the 500-mg strength $(11,12)$.

\section{Isoptin ${ }^{\circledR}$ SR Tablets}

In vitro dissolution data of compositionally proportional 120- and 240-mg Isoptin ${ }^{\circledR}$ SR tablets were generated using three media of $\mathrm{pH} 1.2,4.5$, and 6.8 . Figure 7 shows that the two strengths exhibit similar dissolution behaviors only at $\mathrm{pH}$ 1.2 and failed similarity criteria at both $\mathrm{pH} 4.5$ and 6.8 .

The in vivo performance of the two strengths is evaluated based on a review of bioavailability studies reported in the literature, regulatory filing, and product label.

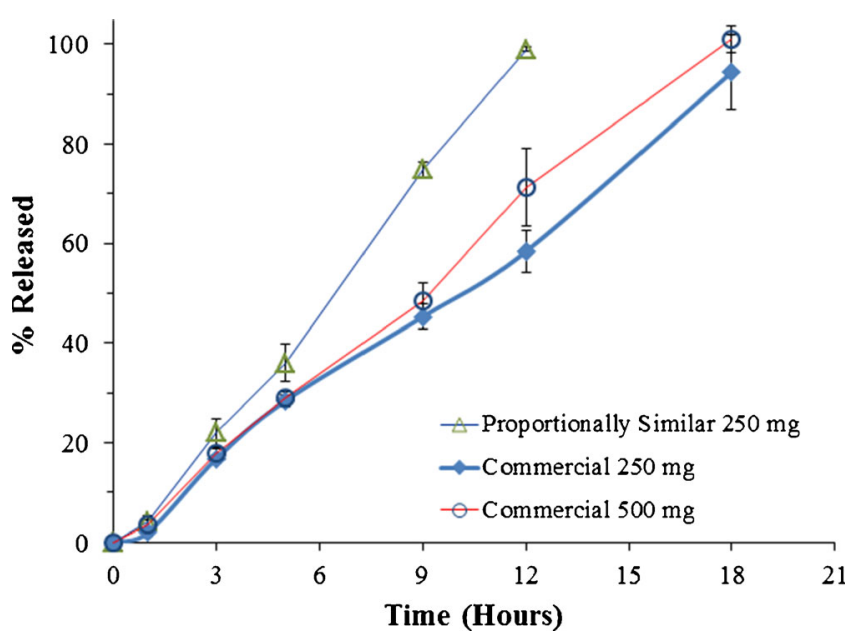

Fig. 3. In vitro dissolution of proportionally similar $\left(f_{2}=39.7\right)$ and reformulated $\left(f_{2}=60.2\right)$ commercial $250-\mathrm{mg}$ ER tablets of drug A compared with the 500-mg ER tablets using an IVIVC-based test method 

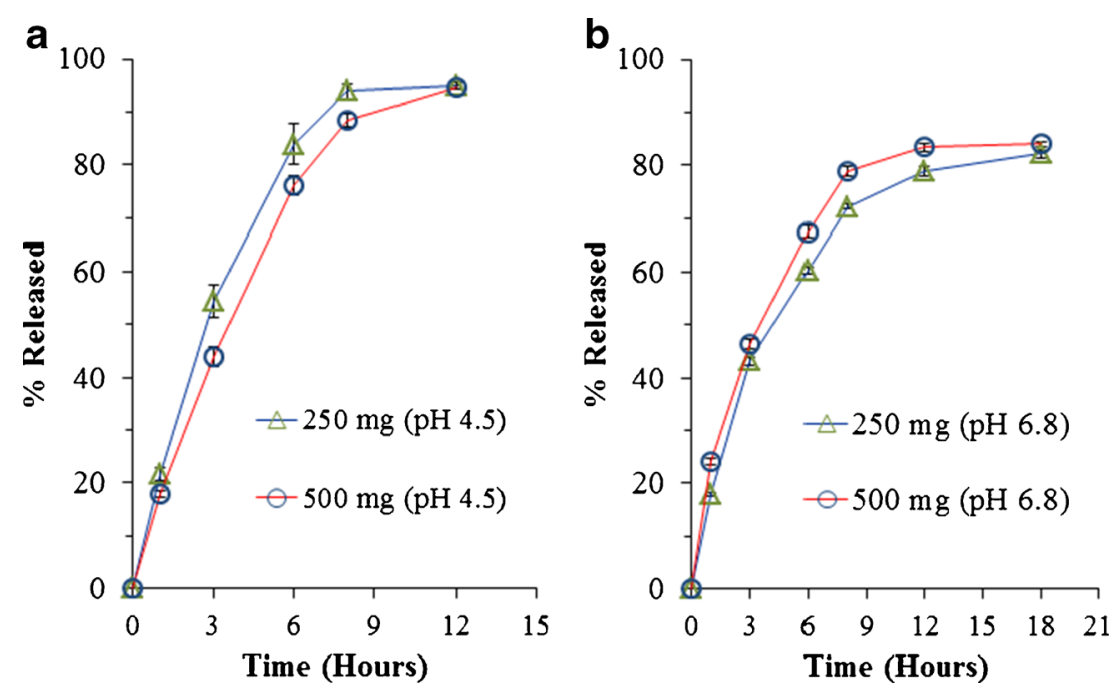

Fig. 4. In vitro dissolution of drug B ER tablets, 250 and $500 \mathrm{mg}$, in multi-pH media. a $\mathrm{pH}$ $4.5\left(f_{2}=60.8\right)$. b pH $6.8\left(f_{2}=63.2\right)$

McEwen et al. reported a crossover multiple-dose pharmacokinetic comparison of Isoptin ${ }^{\circledR}$ SR, 240- and $2 \times 120-\mathrm{mg}$ tablets, in ten healthy volunteers (13). Each regimen was administered once daily for 8 days with a 1-week washout period. No significant differences $(p>0.1)$ in mean $C_{\max }$, $T_{\max }$, and area under the curve (AUC) (0-24) values were observed after dosing two 120-mg tablets and one 240-mg tablet (13). The average $C_{\max }$ and AUC values were 116 and $122 \mathrm{ng} / \mathrm{mL}$ and 1523 and $1416 \mathrm{ng} \mathrm{h} / \mathrm{mL}$ for the two dosing regimens, respectively. Similar results were found for the metabolite, norverapamil. In a separate study comparing two half 240-mg tablets and a single 240-mg tablet, again no statistical differences were observed in average $C_{\max }, T_{\max }$ and AUC (0-24) values, which is consistent with the product label of Isoptin SR $(13,14)$ that allows patients to split the 240-mg tablet in half when necessary. These findings indicate similar in vivo performance between the two proportionally similar strengths despite of the observed differences in in vitro dissolution and tablet sizes.

In summary, investigation of in vitro and in vivo data of four marketed ER products containing different APIs, ratecontrolling polymers, and product designs has shown that all four outcomes illustrated in Fig. 1 are possible. Therefore, in the absence of any known in vitro-in vivo relationship, biowaiver decision of proportionally similar strengths of an ER product based on the in vitro dissolution tests in multimedia lacks adequate scientific basis. It may (1) be acceptable for products that fall within cases II and III, such as drug B ER and drug C ER; (2) lead to unnecessary in vivo bioequivalence study for case I, i.e., products with dissolution
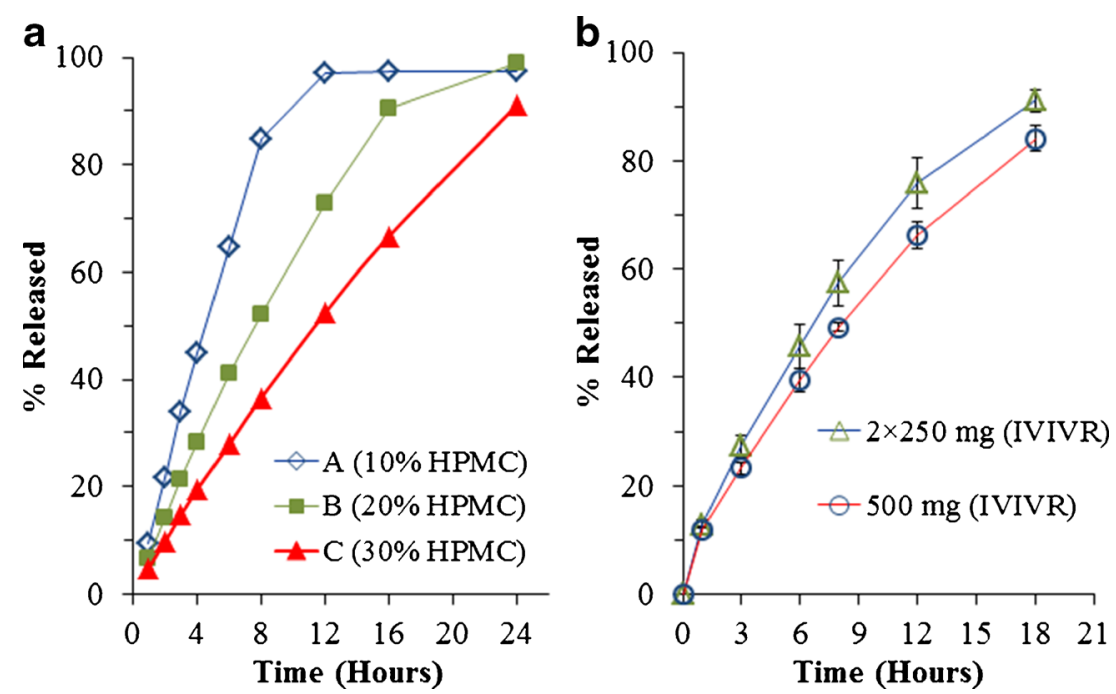

Fig. 5. In vitro dissolution profiles of a three bioequivalent drug B ER tablets containing different levels of HPMC and $\mathbf{b}$ proportionally similar ER tablets, 250 and $500 \mathrm{mg}$, using the IVIVR method 

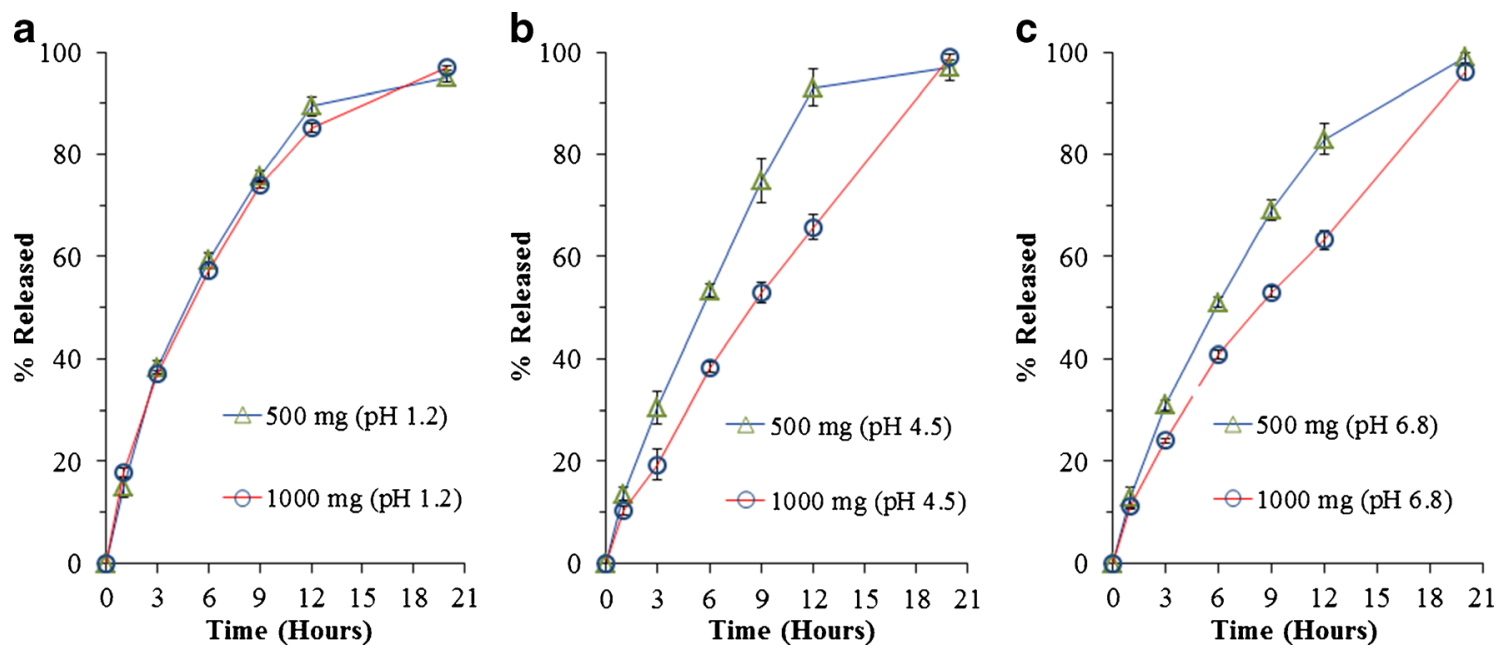

Fig. 6. In vitro dissolution of drug $\mathrm{C}$ ER tablets, 500 and $1000 \mathrm{mg}$, in multi-pH media. a $\mathrm{pH} 1.2\left(f_{2}=78.5\right)$. b $\mathrm{pH} 4.5$ $\left(f_{2}=39.3\right)$. c pH $6.8\left(f_{2}=47.1\right)$

behaviors similar to Isoptin ${ }^{\circledR} \mathrm{SR}$; or (3) result in the approval of certain bioinequivalent products as described in case IV such as proportionally similar lower strength of drug A ER tablet. Thus, establishing an in vitro and in vivo relationship of an ER product is critical for any decision involving the need for conducting bioequivalence studies. In addition, gaining enhanced understanding of how drug properties, product design, and their interplay with test condition influence in vitro dissolution is also important.

\section{DISCUSSION}

\section{Understanding the Influence of Drug Properties and Product Formulation on Drug Release in Multi-pH Media}

Oral extended release systems typically fall into three broad categories: matrix, reservoir, and osmotic systems (4). Depending on drug property and formulation design, drug release from these systems generally involves one or a combination of the following mechanisms: drug diffusion through pores of a barrier, through tortuous channels, or through a viscous gel layer formed by entangled polymer chains; system swelling followed by diffusion and/or erosion and dissolution; or osmotic pressure-induced drug release. For a monolithic dosage form, smaller dosage unit size of the proportionally similar lower strength generally results in a shorter diffusional pathlength and/or time for complete hydration in a matrix system, thinner coating film in the membrane-controlled reservoir, or semipermeable membrane in the osmotic devices because higher $\mathrm{S} / \mathrm{V}$ provides larger normalized surface. As a result, drug release is usually faster than that of the higher strength counterpart.

The design of four ER products in the current study is based on hydrophilic matrix technology which is utilized in over $75 \%$ of the ER products on the market (16) due to its applicability across a broad range of therapeutic drugs and ease of manufacture. They are all single-unit tablets; hence, one might expect similar release rate change between the proportionally similar lower and higher strength across all four products because of their similar increase in $S / V$. However, unlike an inert hydrophobic matrix system that usually involves negligible dimensional changes throughout drug release, drug release from the swellable and erodible matrix system is more complex where the relative contribution of diffusion and polymer erosion to the total drug release not only depends on the properties of specific drug and ratecontrolling polymer, product composition, processing, and dissolution test conditions but may also is constantly changing with time. For drug molecules with negligible solubility, diffusion plays a minimum role and drug release from the matrix is expected to be primarily determined by the rate of surface erosion. Therefore, the increase in drug release rate in this case is generally proportional to the increase in the normalized surface area. For spherical geometry, the increase in $\mathrm{S} / \mathrm{V}$ of a proportionally similar half-strength tablet is approximately $26 \%$. Thus, in vitro drug release from this type of half-strength tablet is generally not expected to pass the dissolution similarity criteria. For soluble drugs or drugs with intermediate solubility, the drug release is much more complex due to the interplay between drug diffusion, particle dissolution, water penetration, polymer hydration that leads to glass-rubber transition (i.e., gel formation and swelling), and the subsequent polymer disentanglement and dissolution from the matrix surface. An added complication is the dynamic aspect of the above processes that changes constantly with time.

The gel layer formation and development is important for drug release from swellable hydrophilic matrices because the gel regulates both diffusion and polymer erosion processes. During dissolution testing, polymer starts to hydrate when in contact with water, where polymer and water can be considered as a solid solution. With the continuous influx of water, the concentration of the polymer in this solid solution decreases over time and eventually reaches a critical point where the glass-rubber transition occurs, leading to the formation of a rubbery gel. This critical point corresponds to a polymer/water composition where the glass transition temperature is the same as the environmental temperature 


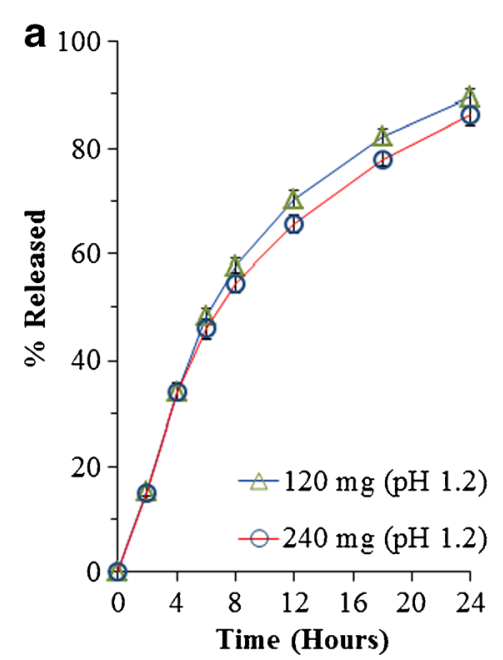

Fig. 7. In vitro dissolution of Isoptin ${ }^{\circledR} \mathrm{SR}$ tablets, 120 and $240 \mathrm{mg}$, in multi-pH media. a pH $1.2\left(f_{2}=73.4\right)$. b pH

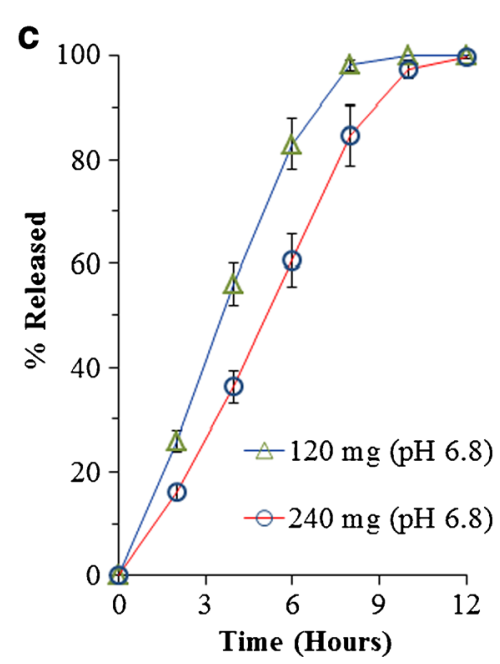

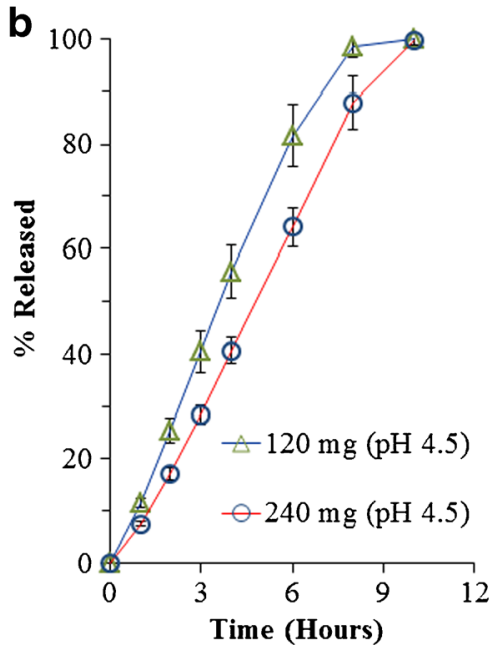

$4.5\left(f_{2}=45.8\right)$. c pH $6.8\left(f_{2}=44.1\right)$ (i.e., $37^{\circ} \mathrm{C}$ ). As more water ingresses through the existing gel, the gel layer thickness would increase over time, leading to an inward movement of the glass-rubber boundary. An outer-toinner concentration gradient of water and inner-to-outer concentration gradient of the polymer are hence created within the gel and the glassy regions. At the gel-water interface, polymer concentration is lowered to a critical point where chain disentanglement occurs and polymer starts to dissolve (i.e., erosion). Therefore, gel layer thickness does not increase indefinitely and a steady state may be reached where polymer erosion balances with water ingression. The development of the gel layer thickness depends on the rate of water diffusion and the rate of polymer erosion. While the rate of water diffusion in the same type of polymeric gel is similar (17), the rate of erosion does change appreciably with molecular weight/viscosity grade (18), polymer chemistry (19), and solubility of the API and other excipients in the matrix. The disentanglement concentration $\left(C_{\mathrm{p}, \mathrm{dis}}\right)$ of HPMC was found to scale up with molecular weight $\left(M_{\mathrm{w}}\right)$ as: $C_{\mathrm{p} \text {,dis }}$ $(\mathrm{g} / \mathrm{mL})=0.05\left(M_{\mathrm{w}} / 96,000\right)^{-0.8}$. For example, Methocel $\mathrm{K} 100 \mathrm{LV}\left(M_{\mathrm{w}}=30 \mathrm{kDa}\right)$ is a lower viscosity grade HPMC, and its disentanglement concentration was measured as $0.13 \mathrm{~g} / \mathrm{mL}$, which is much higher than the $0.05 \mathrm{~g} / \mathrm{mL}$ for Methocel ${ }^{\circledR} \mathrm{K} 4 \mathrm{M}\left(M_{\mathrm{w}}=100 \mathrm{kDa}\right)$. Therefore, insignificant gel layer formation is often visually observed for matrix tablets made with low viscosity grade of HPMC such as K100LV, reflecting a small gel layer thickness that reaches steady state more quickly due to faster polymer erosion. On the contrary, a thick gel layer and significant swelling are noticeable with the matrix tablet prepared using high viscosity grade HPMC such as $\mathrm{K} 15 \mathrm{M}$ and $\mathrm{K} 100 \mathrm{M}$. The slower polymer erosion rate also indicates that gel layer development is more gradual before reaching the steady state, as shown in the simulation in Fig. 8 based on the work of Harland et al. (20).

The gel layer of the slowly eroding polymer matrix increases significantly over the duration of the dissolution testing. The increase in the diffusion pathlength over this period results in decreasing overall drug release if the driving force for drug release remains constant (i.e., similar drug concentration at the glass-rubber boundary). This is essentially similar to drug release from a hydrophobic matrix, where the increasing diffusion pathlength and decreasing surface area over time lead to the square-root time behavior of the overall drug release. For proportionally similar lower and higher strengths, the gel layer thickness is expected to be similar due to the same composition, except at a later stage when complete hydration is reached earlier for the lower strength. Hence, drug release is relatively faster for the lower strength due to its increased S/V. But, when drug solubility is high, the drug molecules will be fully dissolved at the glassrubbery interface and throughout the gel layer. The drug concentration gradient in the gel layer will gradually decrease, reducing release rate over time. Therefore, the overall drug release tends to be affected less by the difference in $\mathrm{S} / \mathrm{V}$. In other words, for slowly erodible matrix tablets of soluble drugs, proportionally similar lower and high strengths will more likely to have similar dissolution profiles. However, if the drug solubility is low such that most of the drug molecules are not solubilized in the gel layer, drug diffusion will play a decreased role, and the concentration gradient of the drug will be more similar between proportionally lower and higher strengths. In this case, the difference in the overall drug release rate will more closely align with the difference in $\mathrm{S} / \mathrm{V}$.

Similar analysis can be applied to matrices prepared using fast eroding polymers. Because of the fast rate of polymer erosion, a thin and steady-state gel layer is developed quickly and maintained throughout the dissolution process until complete hydration of the tablet core. If the drug is fully solubilized, drug release from proportionally similar lower and higher strengths has a higher chance to dissolve similarly, depending on the dose difference between the strengths. For drugs where diffusion is limited by solubility, release rate may be governed by the difference in $\mathrm{S} / \mathrm{V}$. However, the requirement on drug solubility may not be as high as in the cases of slowly eroding polymer in order to 


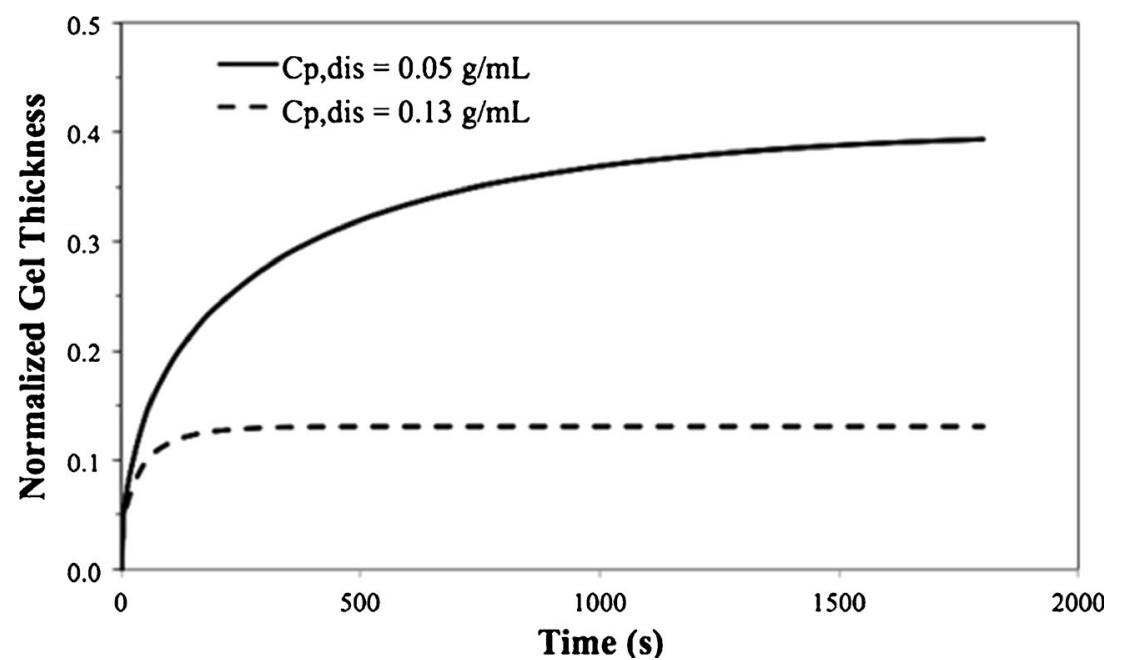

Fig. 8. Impact on gel layer thickness development by the polymer erosion rate

compensate for the disparity in $\mathrm{S} / \mathrm{V}$, because the concentration gradient is over a thinner gel layer (i.e., short diffusion pathlength).

The above analysis indicates that, for high solubility drugs, the release from proportionally similar lower and higher strength hydrophilic matrices tends to be less sensitive to $\mathrm{S} / \mathrm{V}$ than low solubility drugs. In addition, we postulate that the ratio of drug solubility/gel layer thickness is likely a critical parameter impacting the contribution of diffusion to overall drug release. A high solubility/gel thickness ratio will compensate more for the difference in $\mathrm{S} / \mathrm{V}$ between the proportionally similar lower and higher strengths. In addition, whether a lower and a higher strength may have similar dissolution profiles is also dependent on the dose difference which determines the extent of S/V difference. Generally, half-strength tablets result in approximately $20-30 \%$ increase in S/V over its full strength counterpart of the same geometry. Therefore, the lower and higher strength of soluble drugs, in particular, drug with high solubility/gel layer thickness ratio, will likely pass the dissolution similarity test. It should be noted that the properties of other excipients present in significant quantity, and the potential interactions among formulation components usually also play a role during the drug release process, such as the case with Isoptin ${ }^{\circ}$ SR, making the system more complicated.

To help understand how API properties, formulation design, and in vitro test condition may influence the linkage between in vitro and in vivo performance, characteristics of the API and formulation relevant to drug release control and behaviors were evaluated. Information provided in Table I shows that the percent changes of $\mathrm{S} / \mathrm{V}$ of the lower to higher strengths are rather similar across the four tested products (22-34\%), even though the tablet geometry between Isoptin SR 120 and $240 \mathrm{mg}$ is different. Thus, the observed different in vitro behaviors in multimedia dissolution testing across four products are compared based on an integrated analysis of (1) drug $\mathrm{p} K_{\mathrm{a}}$ and solubility; (2) type, grade, and content of polymer; and (3) drug loading and other excipients. To derive the apparent release-controlling mechanism under different test conditions, a well-known semi-empirical approach was used by fitting of the release data to a simple exponential equation (Eq. 2) (21), and the results are provided in Table II.

$M_{t} / M_{\infty}=k t^{n}$

$M_{t}$ and $M_{\infty}$ are the absolute cumulative amount of drug released at time $t$ and infinite time, respectively; $k$ is a constant incorporating structural and geometric characteristics of the device, and $n$ is the release exponent, indicative of the apparent mechanism of drug release.

It has been established that an $n$ value of 0.5 (i.e., square root of time) is indicative of a diffusional mechanism and an $n$ value of 1.0 suggests an erosion mechanism, assuming a film/ slab geometry, whereas an $n$ value of $0.5-1.0$ indicates a combination thereof. The critical values of the exponents are somewhat different for other geometries (21). For example, the critical diffusional exponents are 0.45 and 0.43 for Fickian diffusion from cylinders and spheres, respectively. The corresponding limiting values for erosion-based release are 0.89 and 0.85 , respectively. The critical values are not available for geometries other than the cylinder and sphere (such as the oval tablet in this study). However, they are expected to lie in between those values obtained from cylinder and spheres, given their intermediate aspect ratios.

\section{Case I: Isoptin ${ }^{\circledR}$ SR Tablets}

The rate-controlling polymer of Isoptin ${ }^{\circledR}$ SR is low viscosity grade sodium alginate, a $\mathrm{pH}$-dependent, natural water-soluble linear unbranched polysaccharides consisting of different proportions of $\beta$-D-mannuronic acid (M) and $\alpha$-Lguluronic acid $(\mathrm{G})$ units. At acidic $\mathrm{pH}$ below the $\mathrm{p} K_{\mathrm{a}}$ of $\mathrm{M}$ (3.38) and $\mathrm{G}$ (3.65) monomers, sodium alginate is converted to insoluble and nonswellable alginic acid (22), resulting in altered release-controlling characteristics.

At $\mathrm{pH} 1.2$, the in vitro release from Isoptin ${ }^{\circledR}$ SR tablets is slow and mainly controlled by diffusion because of high solubility of the API and the formation of the inert alginic acid. In addition, the surface area available 
Table I. Characteristics of Drug Substances and Product Design

\begin{tabular}{|c|c|c|c|c|}
\hline Drug product & Drug A & Drug B & Drug $\mathrm{C}$ & Isoptin ${ }^{\circledR}$ SR \\
\hline \multicolumn{5}{|l|}{ Drug properties } \\
\hline $\mathrm{p} K_{\mathrm{a}}$ & 4.8 (acidic) & 8.76 (basic) & 4.86 (acidic); 2.19 (basic) & 8.8 (basic) \\
\hline Solubility at $\mathrm{pH} 1.2$ & $\begin{array}{l}\text { Slightly soluble } \\
\quad(1.3 \mathrm{mg} / \mathrm{mL})^{a}\end{array}$ & N/A & $\begin{array}{l}\text { Freely soluble } \\
\qquad(180 \mathrm{mg} / \mathrm{mL})^{a}\end{array}$ & $\begin{array}{l}\text { Soluble } \\
\quad(60-70 \mathrm{mg} / \mathrm{mL})\end{array}$ \\
\hline Solubility at $\mathrm{pH} 4.5$ & $\begin{array}{l}\text { Slightly soluble } \\
\qquad(2.1 \mathrm{mg} / \mathrm{mL})^{b}\end{array}$ & $\begin{array}{l}\text { Freely soluble } \\
\qquad(230 \mathrm{mg} / \mathrm{mL})^{b}\end{array}$ & Soluble $(24 \mathrm{mg} / \mathrm{mL})^{b}$ & $\begin{array}{l}\text { Soluble } \\
\qquad(60-70 \mathrm{mg} / \mathrm{mL})\end{array}$ \\
\hline Solubility at $\mathrm{pH} 6.8$ & $\begin{array}{l}\text { Freely soluble } \\
\qquad(165 \mathrm{mg} / \mathrm{mL})^{b}\end{array}$ & $\begin{array}{l}\text { Slightly soluble } \\
\qquad(1.2 \mathrm{mg} / \mathrm{mL})^{b}\end{array}$ & Very soluble $(1.4 \mathrm{~g} / \mathrm{mL})^{b}$ & $\begin{array}{l}\text { Sparingly soluble } \\
\quad(\sim 13 \mathrm{mg} / \mathrm{mL})\end{array}$ \\
\hline \multicolumn{5}{|l|}{ Product design } \\
\hline Tablet shape & Oval & Oval & Oval & $\begin{array}{l}\text { Oval }(240 \mathrm{mg}) \\
\text { Round }(120 \mathrm{mg})\end{array}$ \\
\hline$\% \mathrm{~S} / \mathrm{V}^{a}$ & 122 & 132 & 134 & 128 \\
\hline Drug loading & $53 \%$ & $50 \%$ & $83 \%$ & $35 \%$ \\
\hline Polymer & HPMC & HPMC & HPMC & Alginate \\
\hline Viscosity grade & High & Low & High & Low \\
\hline Polymer level & High & Medium & Low & High \\
\hline Soluble filler & Low & High & None & None \\
\hline
\end{tabular}

${ }^{a} \mathrm{~S} / \mathrm{V}$ surface area-to-volume ratio of lower strength normalized to that of the higher strength, N/A not available, HPMC hydroxyl methylcellulose

${ }^{b}$ Estimated based on $\mathrm{pH}$-solubility profile, intrinsic solubility, and $\mathrm{p} K_{\mathrm{a}}$

for drug release also increases over time due to tablet cracking and lamination at acidic $\mathrm{pH}(23,24)$. Hence, a $28 \%$ change in $\mathrm{S} / \mathrm{V}$ of the $120-\mathrm{mg}$ strength showed negligible influence on drug release rate compared with the 240-mg strength. At $\mathrm{pH} 4.5$ and 6.8, sodium alginate hydrates and swells rapidly, forming a gel layer surrounding the tablet. Unlike matrices consisting of low viscosity grade HPMC, the gel layer development of alginate is significant $(23,24)$. However, it is interesting to note that drug release at $\mathrm{pH} 4.5$ was essentially governed by polymer erosion in spite of high drug solubility. In addition, drug release profiles are essentially the same as those at $\mathrm{pH} 6.8$ even though drug solubility is drastically lower at high $\mathrm{pH}$. These observations can be attributed to the interaction between the protonated tertiary amine cation of verapamil and the carboxyl anion of sodium alginate, resulting in erosion-controlled drug release and a decrease in free drug concentration in the gel. As a result, the release rate at $\mathrm{pH} 4.5$ and 6.8 is influenced by the difference in $\mathrm{S} / \mathrm{V}$ between the two proportionally similar strengths, resulting in dissimilar dissolution profiles at both $\mathrm{pH}$ values. Therefore, in assessing proportionally similar Isoptin ${ }^{\circledR}$ SR tablets, in vitro multimedia dissolution testing is found to be overdiscriminating with respect to their in vivo performance because the two strengths have been shown to be bioequivalent.

\section{Case II: ER Tablets of Drug B}

Drug B is freely soluble at $\mathrm{pH}$ 4.5. The rate-controlling polymer is low viscosity grade HPMC, Methocel 100LV. Because of the fast erosion rate, a thin, steady-state gel layer

Table II. Fitting of Multimedia Dissolution Testing Data

\begin{tabular}{|c|c|c|c|c|c|c|c|c|}
\hline \multirow{2}{*}{$\frac{\text { Drug product }}{\text { Strength }}$} & \multicolumn{2}{|c|}{ ER product $A$} & \multicolumn{2}{|c|}{ ER product $B$} & \multicolumn{2}{|c|}{ ER product $\mathrm{C}$} & \multicolumn{2}{|c|}{ Isoptin $®$ SR } \\
\hline & $500 \mathrm{mg}$ & $250 \mathrm{mg}$ & $500 \mathrm{mg}$ & $250 \mathrm{mg}$ & $1000 \mathrm{mg}$ & $500 \mathrm{mg}$ & $240 \mathrm{mg}$ & $120 \mathrm{mg}$ \\
\hline \multicolumn{9}{|l|}{$\mathrm{pH} 1.2$} \\
\hline$n$ & 0.66 & 0.71 & - & - & 0.65 & 0.74 & 0.54 & 0.57 \\
\hline$k$ & 3.82 & 4.75 & - & - & 17.89 & 15.50 & 16.78 & 16.53 \\
\hline$R^{2}$ & 0.99 & 1.00 & - & - & 1.00 & 0.99 & 0.97 & 0.98 \\
\hline$f_{2}$ & 51.4 & & - & & 78.5 & & 73.4 & \\
\hline \multicolumn{9}{|l|}{$\mathrm{pH} 4.5$} \\
\hline$n$ & 0.51 & 0.46 & 0.80 & 0.76 & 0.76 & 0.77 & 1.20 & 1.10 \\
\hline$k$ & 9.68 & 13.93 & 18.4 & 22.23 & 9.64 & 13.41 & 7.52 & 11.85 \\
\hline$R^{2}$ & 1.00 & 1.00 & 1.00 & 0.99 & 0.99 & 1.00 & 1.00 & 1.00 \\
\hline$f_{2}$ & 54.1 & & 60.8 & & 39.3 & & 45.8 & \\
\hline \multicolumn{9}{|l|}{$\mathrm{pH} 6.8$} \\
\hline$n$ & 0.49 & 0.49 & 0.58 & 0.69 & 0.71 & 0.76 & 1.15 & 0.98 \\
\hline$k$ & 14.56 & 16.44 & 24.22 & 18.52 & 11.16 & 13.15 & 7.39 & 13.69 \\
\hline$R^{2}$ & 1.00 & 0.99 & 1.00 & 0.98 & 1.00 & 1.00 & 1.00 & 1.00 \\
\hline$f_{2}$ & 61.0 & & 63.2 & & 47.1 & & 44.1 & \\
\hline
\end{tabular}


is established quickly and maintained throughout the drug release. Therefore, the release profile is mostly linear, reflecting an apparent mechanism of polymer erosion ( $n=0.76$ and 0.80 for the 250- and 500-mg strengths, respectively) However, high solubility tends to compensate for the difference in $\mathrm{S} / \mathrm{V}$ due to the relatively high drug solubility/gel thickness ratio, and hence, the two strengths exhibit similar dissolution profiles $(f=60.8)$. The solubility is much lower at pH $6.8(\sim 1.2 \mathrm{mg} / \mathrm{mL})$, which would generally sugget increased influence by $\mathrm{S} / \mathrm{V}$ on drug release. The observed curvature and filtted release exponenets of 0.69 and 0.58 for the $250 \mathrm{mg}$ and $500 \mathrm{mg}$ strengths, respectively, can be attributed to non-sink conditions and incomplete drug release due to lower solubility. As a result, slow dissolution of the drug particles upon their releaese from the tablets plays a significant role that negates the impact of the $32 \%$ increase in $\mathrm{S} / \mathrm{V}$, resulting in a similar dissolution profile at $\mathrm{pH}$ 6.8. In addition, the highly soluble filler in the formulation can also create pockets in the weakened gel layer, making the drug release a more complex process.

When utilizing the test method developed for mapping in vitro and in vivo performance, the 250- and 500-mg strengths exhibit similar drug release (Fig. 5b), suggesting similar in vivo performance. Thus, for ER tablets of drug $\mathrm{B}$, the in vitro multimedia dissolution testing fortuitously turns out to be useful for comparing the proportionally similar strengths.

\section{Case III: ER Tablets of Drug C}

A high viscosity grade HPMC, Methocel E10M is employed in drug C ER tablet. During dissolution, the gel layer thickness development is very pronounced. Drug $\mathrm{C}$ is a zwitterion with an acidic $\mathrm{p} K_{\mathrm{a}}$ of 4.75 and a basic $\mathrm{p} K_{\mathrm{a}}$ of 1.2 and has a U-shaped $\mathrm{pH}$-solubility profile over the $\mathrm{pH}$ range of $1.2-6.8$. Solubility at $\mathrm{pH} 1.2$ is very high $(\sim 180 \mathrm{mg} / \mathrm{mL})$, and drug release is predominantly diffusion through the gel layer. The high solubility and slower polymer erosion compensated for the $34 \%$ difference in $\mathrm{S} / \mathrm{V}$ between the two proportionally similar strengths and resulted in similar dissolution profiles $\left(f_{2}=78.5\right)$. At $\mathrm{pH} 4.5$, erosion is the predominant mechanism due to low solubility (Fig. 6b). However, it is surprising to observe that at $\mathrm{pH} \mathrm{6.8,} \mathrm{the}$ apparent drug release not only follows a near-zero-order kinetics but also is slower than that at $\mathrm{pH} 1.2$ despite of higher drug solubility $(\sim 1.4 \mathrm{~g} / \mathrm{mL})$. For example, release of the $1000-\mathrm{mg}$ tablet at $6 \mathrm{~h}$ is about $60 \%$ at $\mathrm{pH} 1.2$ and but only about $40 \%$ at $\mathrm{pH} 6.8$. Additionally, the differences in release rates between $\mathrm{pH} 4.5$ and 6.8 media are insignificant even though drug solubility is significantly higher at $\mathrm{pH} 6.8$. These observations suggest a likely interaction between drug $\mathrm{C}$ and HPMC at $\mathrm{pH}$ around and above its acidic $\mathrm{p} K_{\mathrm{a}}$ where the carboxylic anion is the dominant species. The interaction may lead to the formation of a certain type of drug/HPMC complex, significantly reducing free drug concentration in the gel layer for diffusion. Therefore, drug release is dictated by polymer matrix erosion. Although the exact mechanism is undefined, hydrophobic interactions between HPMC and certain drug molecules containing carboxylic group such as ibuprofen sodium have been reported in the literature (25). Consequently, when compared with the 1000-mg strength, a 34\% increase in S/ $\mathrm{V}$ of the 500-mg strength led to a significant increase (30$40 \%$ ) in release rate at $\mathrm{pH} 4.5$ and 6.8 , likely due to decreased free drug concentration in the gel layer. Therefore, in evaluating proportionally similar ER drug $\mathrm{C}$ tablets, the dissolution profiles happened to be dissimilar at $\mathrm{pH} 4.5$ and 6.8 , leading to the coincidental agreement with the in vivo data.

\section{Case IV: ER Tablets of Drug A}

Drug A ER tablets contain a high content of high viscosity grade HPMC that erodes slowly and exhibits a significant gel layer development phase. During dissolution, there is a very high degree of polymer swelling. At $\mathrm{pH} 1.2$, the in vitro drug release of the proportionally similar strengths is extremely slow primarily due to low solubility and nonsink condition. The apparent increase of the release rate from 18 to $24 \mathrm{~h}$ observed for the $250-\mathrm{mg}$ strength (Fig. 2a) may result from earlier completion of tablet hydration due to its smaller size. Drug solubility increases at $\mathrm{pH} 4.5$ and much more significantly at $\mathrm{pH}$ 6.8. But it is still inadequate to allow complete dissolution of the solid API in the gel layer. Hence, diffusion through the gel layer is the predominant drug release mechanism as indicated by release exponent values of approximately 0.5 . The increasing gel layer thickness over time due to rapidly swelling and slowly eroding polymer compensated for the $34 \%$ difference in $\mathrm{S} / \mathrm{V}$ between the two proportionally similar strengths and resulted in similar dissolution profiles.

The in vitro results in multimedia differ significantly in both release rate and kinetics from the in vivo apparent absorption profiles obtained by deconvolution $(1,26)$. Using the IVIVC-based test method, the proportionally similar strengths exhibited not only dissimilar profiles but also a more rapid and predominantly erosion-controlled release mechanism, consistent with the in vivo observation (Fig. 3). Consequently, a change in $\mathrm{S} / \mathrm{V}$ ratio resulted in different release rates between the two strengths. The results of the current study indicate that in vitro multimedia dissolution testing is unable to discriminate between formulations with acceptable and unacceptable in vivo behavior of drug A ER tablets, which is consistent with previous studies involving formulations with different release rates during the development of IVIVC (26).

\section{Evaluating Implications of the Study Findings on Biowaiver Requirement}

Four cases shown in Fig. 1 are represented by four model ER products used in the study. Among them, only case II (drug B ER) and case III (drug C ER) comply with the biowaiver requirements stipulated in regulation from the perspective of assured product quality and performance. Case I (Isoptin SR) presents a scenario where biowaiver of proportionally similar strengths that are bioequivalent would be rejected because in vitro testing using multi-pH media is overdiscriminating. This case would generally result in a bioequivalence study without presenting risk to patients except for unnecessary exposure 
of the trial subjects to the test drug and cost and resources associated with the study. However, case IV (drug A ER) represents a situation that could have the most serious impact on product efficacy and safety or could even cause patient harm, particularly for ER dosage forms containing drugs with NTI. For instance, bioinequivalent strengths of antiepileptic products, such as Depakote ER, Keppra XR, and Tegretol XR, could lead to the increased risk of adverse events and even breakthrough seizures due to incorrect dose titration. A recent example of case IV was related to the withdrawal of regulatory approval in 2012 by the FDA of the $300-\mathrm{mg}$ strength of Budeprion XL, a bupropion hydrochloride ER tablet. Since it was first approved in 2006, the product had been the subject of many reports of reduced efficacy when a switch was made from the innovator's Wellbutrin XL 300-mg tablets. The 300-mg strength was originally approved through a biowaiver based on comparative in vitro study with the 150-mg strength. It was later determined to be not bioequivalent in both the rate and extent of absorption to the reference product in a study commissioned by the FDA (27). Subsequently, four other generic ER tablets of bupropion hydrochloride were subject to in vivo bioequivalence studies per request from the FDA because all of them were originally granted waivers of in vivo $\mathrm{BE}$ testing on the 300-mg strength (28). As a result, one additional $300-\mathrm{mg}$ strength product was withdrawn from the market due to bioinequivalence. A review of ER technologies utilized in four branded and five generic ER tablets of bupropion $\mathrm{HCl}$ indicates diverse mechanisms for controlling drug release that include hydrophilic and hydrophobic matrices, reservoir system, and matrix-reservoir combination. Bupropion is a weak base with $\mathrm{pH}$-dependent solubility. Mechanistically, the difference in $\mathrm{S} / \mathrm{V}$ between the single-unit compositionally proportional strengths is likely to affect drug release from all types of ER systems employed in these products as discussed in the previous section. Whether changes in drug release are manifested in the in vitro multimedia testing or in vivo absorption are primarily determined by the drug properties, formulation design, dosage form geometry, and their interplays with in vitro test condition and in vivo absorption environment. This is why strength-specific formulations are required to achieve bioequivalence for certain products while proportionally similar formulations are found bioequivalent between different strengths for others.

For over two decades, guidelines issued by the leading regulatory agencies and organizations, such as the FDA, EMA, and World Health Organization (WHO), have been allowing biowaiver of proportionally similar lower strengths of extended release drug products based on in vitro multimedia dissolution testing. The present study has shown that the scientific rationale for this specific part of regulation is inadequate. First of all, there is no known evidence that similar in vitro drug release obtained in the multi-pH media using standard USP apparatus has the general ability to reliably predict or assure equivalent in vivo performance for any type of proportionally similar ER products containing any APIs, particularly for single-unit dosage forms. Additionally, for formulations containing API and rate-controlling materials of which solubility is independent of $\mathrm{pH}$, multimedia dissolution testing is not different from testing in single medium because drug release profiles usually remain unchanged within the biological $\mathrm{pH}$ range. Secondly, the in vitro drug release behaviors of ER dosage forms are highly dependent on the characteristics of specific API, rate-controlling polymer used, delivery technology, and dosage form designs as well as the interplays between these variables and the dissolution test method and conditions. Thus, a possible impact of $\mathrm{S} / \mathrm{V}$ change of proportionally similar ER dosage forms on product performance in vitro and in vivo should be evaluated based on the understanding of the drug substance, drug product, specific test method, and condition on a case-by-case basis. To assure consistent in vivo performance, the current regulation regarding biowaiver of proportionally similar strengths of any ER products solely based on the in vitro tests should be reassessed. In the absence of a proven relationship between the in vitro and in vivo data, bioequivalence study should be required for approving different strengths of any monolithic ER dosage forms except for dosage forms of which strengths are proportionally similar with negligible S/V change (e.g., low drug loading dosage forms) or differ only in the number of dosage unit (e.g., multiunit dosage form).

\section{CONCLUSIONS}

Existing guidelines issued by the major regulatory bodies accept biowaiver of proportionally similar strengths of ER drug products based on matching in vitro release profiles generated in multimedia provided different strengths are of the same dosage form with the same release mechanism and linear pharmacokinetics. However, the relationship between in vitro behaviors and in vivo performance of ER drug products is complex and dependent highly on many variables related to drug substance, product design, formulation, test method, and their interplays. There is no general link between the in vivo apparent drug absorption taking place in the dynamic environment of the gastrointestinal tract and in vitro data generated under the static condition of the standard USP dissolution apparatus. Through the investigation of in vitro and in vivo performances of four marketed ER dosage forms, this study (1) offers insight into how interplays among the API, formulation, and dissolution media may affect in vitro behavior and its uncertain link to in vivo performance, and (2) shows that there is insufficient scientific basis for the current regulation that permits biowaiver of proportionally similar strengths of ER drug products. Therefore, the biowaiver requirements need to be reevaluated. It is in the best interests of patients to consider changing the requirement to only apply to proportionally similar formulations with no or minimum change in size and dimension and multiple-unit dosage forms, such as pellets, beads, or mini-tablets. For other types of ER dosage forms, a bioequivalence study should be required to assure product quality, efficacy, and safety unless an in vitro and in vivo relationship is established. 


\section{ACKNOWLEDGMENTS}

The authors would like to acknowledge Ms. Judie Reynolds at AbbVie, Inc. for assistance in conducting experiments.

\section{COMPLIANCE WITH ETHICAL STANDARDS}

Disclosures AbbVie funded the study and participated in the interpretation, review of data, and approval of publication. Yihong Qiu and Deliang Zhou are AbbVie employees. Zhongqiang Lin is a graduate student and Stephen Hoag is employed at the School of Pharmacy, University of Maryland.

\section{REFERENCES}

1. Dutta S, Qiu Y, Samara E, Cao G, Granneman GR. Once-a-day extended-release dosage form of divalproex sodium III: development and validation of a level A in vitro-in vivo correlation (IVIVC). J Pharm Sci. 2005;94(9):1949-56.

2. Guidance for Industry Bioavailability and Bioequivalence Studies Submitted in NDAs or INDs-general considerations DRAFT GUIDANCE. In: CDER, FDA, editors. 2014.

3. Guidance for Industry Bioavailability and Bioequivalence Studies for Orally Administered Drug Products-general considerations Rev 1. In: CDER, FDA, editors. Mar 2003.

4. Yihong Qiu YC, Geoff G, Zhang Z. Developing solid oral dosage forms: pharmaceutical theory and practice. Elsevier; 2009.

5. Pharmacopeia US. Buffer solutions. USP Official Monograph 36 : USP Official Monograph 36; 2013.

6. Pharmacopeia US. Divaproex sodium extended-release tablets. USP Official Monograph 362013.

7. Pharmacopeia US. Niacin extended-release tablets. USP Official Monograph 36: USP Official Monograph 36; 2013.

8. Pharmacopeia US. Verapamil hydrochloride extended release tablets. Second Supplement to USP 36-NF31; 2014.

9. Pharmacopeia US. Clarithromycin extended-release tablets. USP Official Monograph 36; 2013.

10. Notario GF, Palmer RN, Hom RC, Zhang J, Devcich KJ, Semla SJ. Inventors; Abbott Laboratories, assignee. Extended release formulations of erythromycin derivatives. USA patent US006551616B1. 2003.

11. Lauring B, Rosko K, Luo W-L, Wenning L, Kissling J, Roupe K, et al. A comparison of the pharmacokinetics of two different formulations of extended-release niacin. Curr Med Res Opin. 2009;25(1):15-22.

12. Rocca JG, Cefali E, Zhu Y, inventors; Abbott Laboratories, assignee. Low flush niacin formulation. USA patent US20090069275A1. 2008.
13. McEwen J, Durnin C, McMurdo ME, Moreland TA. Sustained-release verapamil: multiple-dose pharmacokinetic comparison of $120-\mathrm{mg}$ and $240-\mathrm{mg}$ tablets and the effect of halving a 240-mg tablet. J Cardiovasc Pharmacol. 1989;13 Suppl 4:S57-9. S57-9.

14. Abbott Laboratories L. Product monograph: ISOPTIN SR verapamil hydrochloride sustained-release tablets $120 \mathrm{mg}, 180$ $\mathrm{mg}$ and $240 \mathrm{mg} 2014$.

15. Erah PO, Goddard AF, Barrett DA, Shaw PN, Spiller RC. The stability of amoxycillin, clarithromycin and metronidazole in gastric juice: relevance to the treatment of Helicobacter pylori infection. J Antimicrob Chemother. 1997;39(1):5-12.

16. Colorcon. Hydrophilic matrix tablets http://www.colorcon.com/ products-formulation/all-products/polymers-controlled-release/ hydrophilic-matrix-tablets.

17. Gao P, Fagerness PE. Diffusion in HPMC gels. I. Determination of drug and water diffusivity by pulsed-field-gradient spin-echo NMR. Pharm Res. 1995;12(7):955-64.

18. Ju RTC, Nixon PR, Patel MV, Tong DM. Drug release from hydrophilic matrices. 2. A mathematical model based on the polymer disentanglement concentration and the diffusion layer. $\mathrm{J}$ Pharm Sci. 1995;84(12):1464-77.

19. Zhou D, Law D, Reynolds J, Davis L, Smith C, Torres JL, et al. Understanding and managing the impact of HPMC variability on drug release from controlled release formulations. J Pharm Sci. 2014;103(6):1664-72.

20. Harland R, Gazzaniga A, Sangalli ME, Colombo P, Peppas N. Drug/polymer matrix swelling and dissolution. Pharm Res. 1988;5(8):488-94.

21. Siepmann J, Peppas NA. Modeling of drug release from delivery systems based on hydroxypropyl methylcellulose (HPMC). Adv Drug Deliv Rev. 2001;48(2-3):139-57.

22. Chan LW, Ching AL, Liew CV, Heng PWS. Mechanistic study on hydration and drug release behavior of sodium alginate compacts. Drug Dev Ind Pharm. 2007:33(6):667-76.

23. Ching AL, Liew CV, Heng PWS, Chan LW. Impact of crosslinker on alginate matrix integrity and drug release. Int J Pharm. 2008;355(1-2):259-68.

24. Mandal S, Basu S, Sa B. Sustained release of a water-soluble drug from alginate matrix tablets prepared by wet granulation method. AAPS PharmSciTech. 2009;10(4):1348-56.

25. Wells ML, Williams SO, Sanftleben RA, Balik SB, Evans BA. Investigation into the dissolution rate increase on storage of Wellbutrin SR 100 mg tablets. AAPS PharmSciTech. 2010;11:113-9.

26. Qiu Y, Garren J, Samara E, Cao G, Abraham C, Cheskin HS, et al. Once-a-day controlled-release dosage form of divalproex sodium II: development of a predictive in vitro drug release method. Journal of Pharmaceutical Sciences. 2003;92(11):231725.

27. FDA. Update: bupropion hydrochloride extended-release 300 mg bioequivalence studies: FDA; 2013 [updated 10-10-2013]. Available from: http://www.fda.gov/drugs/drugsafety/ postmarketdrugsafetyinformationforpatientsandproviders/ ucm322161.htm

28. FDA. FDA/CDER to Valeant Pharmaceuticals International, Inc. (Covington \& Burling) - partial approved and denied docket No. FDA-2012-P-1091. http://www.regulations.gov/!documentDetail;D=FDA-2012-P-1091-00042013. 